\title{
Extraction of $\alpha$-mangostin from Garcinia mangostana L. using alternative solvents: Computational predictive and experimental studies
}

\author{
Kunnitee Bundeesomchok ${ }^{\mathrm{a}}$, Aurore Filly ${ }^{\mathrm{c}}$, Njara Rakotomanomana ${ }^{\mathrm{c}}$, \\ Pharkphoom Panichayupakaranant ${ }^{\mathrm{a}, \mathrm{b},{ }^{* *} \text {, Farid Chemat }}{ }^{\mathrm{c}, *}$ \\ ${ }^{a}$ Department of Pharmacognosy and Pharmaceutical Botany, Faculty of Pharmaceutical Sciences, Prince of Songkla University, Hat-Yai, Songkhla 90112, \\ Thailand \\ ${ }^{\mathrm{b}}$ Phytomedicine and Pharmaceutical Biotechnology Excellence Center, Faculty of Pharmaceutical Sciences, Prince of Songkla University, Hat-Yai, Songkhla \\ 90112, Thailand \\ ${ }^{c}$ Avignon University, INRA, UMR408, GREEN Extraction Team, F-84000 Avignon, France
}

Keywords:

Green solvents

Extraction

Garcinia mangostana

Hansen

COSMO-RS

\begin{abstract}
A B S T R A C T
This study evaluated the performance of alternative green solvents, i.e. $D$-limonene, dimethylcarbonate (DMC), ethanol, ethyl acetate, ethyl lactate and methyltetrahydrofuran (MeTHF) compared to the petroleum based dichloromethane, for extraction of $\alpha$-mangostin from Garcinia mangostana pericarps. The Hansen solubility parameters (HSPs) were used to explain the dissolution behavior of the solutes and solvents, and the conductor-like screening model for realistic solvation. The (COSMO-RS), a statistical thermodynamic approach based on the results of quantum chemical calculations for comprehending the dissolving mechanisms were used to predict the extraction prediction. On the basis of the Hansen analysis, dichloromethane was the most suitable solvent for extraction of $\alpha$-mangostin. However, COSMO-RS analysis showed a higher solubility of $\alpha$-mangostin in ethyl lactate, DMC, MeTHF, ethyl acetate and ethanol. Moreover, the experimental studies using a classical reflux extraction followed by a quantitative HPLC analysis of $\alpha$-mangostin showed similar results to the predictive values from the COSMO-RS model. The $\alpha$-mangostin levels extracted by ethyl lactate, DMC, MeTHF, ethyl acetate and ethanol were higher than those using dichloromethane and $D$-limonene. The results support the potential use of ethyl lactate, DMC, MeTHF, and ethanol as alternative green solvents for the preparation of $\alpha$-mangostin extracts.
\end{abstract}

\section{Introduction}

Garcinia mangostana L. (mangosteen) is an important economic plant grown in Eastern and Southern Thailand. The fruits are popularly known as the "queen of fruits", as they are produce a popular refreshing tasty juice. The fruit pericarps, are also known for their ethno-medicinal values and these are a by-product of the mangosteen juice factory. G. mangostana pericarp extracts are enriched in xanthones, a group of bioactive compounds (Zarena \&

\footnotetext{
* Corresponding author.

** Corresponding author. Faculty of Pharmaceutical Sciences, Prince of Songkla University, Hat-Yai, Songkhla 90112, Thailand.

E-mail addresses: pharkphoom.p@psu.ac.th (P. Panichayupakaranant), farid chemat@univ-avignon.fr (F. Chemat).
}

Sankar, 2011). The major compounds in the extracts are prenylated xanthones, particularly $\boldsymbol{\alpha}$-mangostin (Nualkaew et al., 2012). This has been used as a standard marker for their standardization. Extracts from G. mangostana pericarps have been reported for their various pharmacological activities, including their actions as antioxidants, and their anticancer (Moongkarndi et al., 2014), antimicrobial (Sakagami, Iinuma, Piyasena, \& Dharmaratne, 2005), antiinflammatory (Cui et al., 2010), and wound healing (Nganlasom, Suttitum, Jirakulsomchok, \& Puapairoj, 2008) properties. The extracts of $G$. mangostana pericarps have also been used in several commercial nutritional supplements, pharmaceutical products and cosmetics (Pothitirat, Chomnawang, \& Gritsanapan, 2009). It has been reported that dichloromethane was the most suitable solvent for extraction of $\alpha$-mangostin from G. mangostana pericarps (Pothitirat, Chomnawang, \& Gritsanapan, 2010). However, the use 
Version définitive du manuscrit publiée dans / Final version of the manuscript published in :

LWT - Food Science and Technology (2016), Vol. 65, p. 297-303, DOI: 10.1016/j.Iwt.2015.08.036

Journal homepage : www.elsevier.com/locate/lwt

of dichloromethane, a halogenated hydrocarbon as a solvent for extraction is restricted due to its toxicities (CalEPA, 2000), and limitations on its use in the development of herbal products.

Nowadays, industrial regulations require a diminished consumption of petrochemical solvents and volatile organic compounds. Moreover, the absence of risk during extraction and the safety of the ingredients used are a major concern and have drawn attention towards the need to use greener solvents (Rombaut, Fabiano-Tixier, Bily, \& Chemat, 2014). Alternative green solvents, a renewable resource produced from biomasses such as wood, starch, vegetable oils or fruits are high power, biodegradable, low toxicity and low flammability. Extensive studies have shown the importance and the reported potential of green solvents that could be alternatively used instead of the petrochemical solvents, e.g. extraction of fat and oils from rapeseed using MeTHF instead of hexane (Sicaire et al., 2015), and extraction of oil from microalgae using $D$-limonene instead of hexane (Tanzi, Abert-Vian, Ginies, Elmaataoui, \& Chemat, 2012). Recently, the dissolving power and selectivity of solvents can be theoretically predicted using computational programs. Currently two computational programs, i.e. the Hansen solubility parameters (HSPs) and a conductor like screening model for realistic solvation (COSMO-RS) are being used to predict the most suitable solvents for extraction of natural products (Filly et al., 2014; Filly, Fabiano-Tixier, Fernandez, \& Chemat, 2015; Li, Fabiano-Tixier, Ginies, \& Chemat, 2014).

The aim of this work was to evaluate the ability of some alternative 'green' solvents to extract $\alpha$-mangostin compared to dichloromethane using the computational programs and experimental extractions (Fig. 1). The computational predictive methods were performed via an HSP program that explained the dissolution behavior of solutes and solvents, and the COSMO-RS program, a statistical thermodynamics approach based on the result of quantum chemical calculations that provided explanations of the mechanisms of dissolution. The results from these predictive computational methods were confirmed by those from experimental extractions.

\section{Materials and methods}

\subsection{Plants material}

Fresh mature G. mangostana fruits were from a market in the Hat-Yai district, Songkhla province, Thailand. The fruits of G. mangostana were cleaned and the edible parts were removed. The pericarps were cut into small pieces, and dried at $50{ }^{\circ} \mathrm{C}$ for $72 \mathrm{~h}$ in a hot air oven. The dried pericarps were then ground into a fine powder using a grinder, and passed through a sieve No. 45 . The dried pericarp powder was kept in a well-closed container and protected from light until further use.

\subsection{Reagents}

The solvents used were analytical grade. Dichloromethane (purity 99.9\%) was from Merck, Germany. Ethyl acetate (purity 99\%) and (R)-(+)-limonene (purity 93\%) were from Sigma Aldrich, Germany. Ethanol (purity 100\%) and 2-methyltetrahydrofuran (2MeTHF) (purity 99\%) were from VWR International, Germany. Ethyl lactate (purity 99\%) and dimethylcarbonate (DMC) (purity 99\%) were from Alfa Aesar, Germany. A sample of $\alpha$-mangostin was purified and identified by the method described by Pothitirat et al. (2010).

\subsection{Computational methods: HSPS}

Solubility parameters for predicting the solubility of a solute was proposed by Hansen (Hansen, 1969). HSPs are based on the concept that the total cohesive energy density is approximated by the sum of the energy densities required to overcome atomic dispersion forces $\left(\delta_{\mathrm{d}}^{2}\right)$, molecular polar forces arising from dipole moments $\left(\delta_{\mathrm{p}}^{2}\right)$ and hydrogen-bonds between molecules $\left(\delta_{\mathrm{h}}^{2}\right)$, as given in the following equation.

$\delta_{\text {total }}^{2}=\delta_{\mathrm{d}}^{2}+\delta_{\mathrm{p}}^{2}+\delta_{\mathrm{h}}^{2}$

Where $\delta_{\text {total }}$ is the Hansen total solubility parameter, which consists of three HSPs in terms of dispersion $\left(\delta_{\mathrm{d}}\right)$, polar $\left(\delta_{\mathrm{p}}\right)$ and hydrogen bonding $\left(\delta_{\mathrm{h}}\right)$.

For HSP solvent optimization, a simple composite affinity parameter, the relative energy difference (RED) number, has been calculated using Equation (2) to determine the solubility between solvent and solute.

$\mathrm{RED}=\mathrm{R}_{\mathrm{a}} / \mathrm{R}_{\mathrm{O}}$

Where $R_{0}$ is the radius of a Hansen solubility sphere, and $R_{a}$ is the distance of a solvent from the center of the Hansen solubility sphere, given by Equation (3):

$\mathrm{R}_{\mathrm{a}}^{2}=4\left(\delta_{\mathrm{d}} \mathrm{A}-\delta_{\mathrm{d}} \mathrm{B}\right)^{2}+\left(\delta_{\mathrm{p}} \mathrm{A}+\delta_{\mathrm{p}} \mathrm{B}\right)^{2}\left(\delta_{\mathrm{h}} \mathrm{A}-\delta_{\mathrm{h}} \mathrm{B}\right)^{2}$

Where A refers to the solute and B refers to the solvent.

The factor 4 in Eq. (3), based on Prigogine's Corresponding States Theory, has proved to effectively expand the dimensions in order to give spherical plots. In general, the parameter follows the classical "like dissolves like" rule: the smaller $\mathrm{R}_{\mathrm{a}}$ is the greater affinity between solute and solvent. It means that good potential solvents have an RED of less than 1 (the compound has similar properties

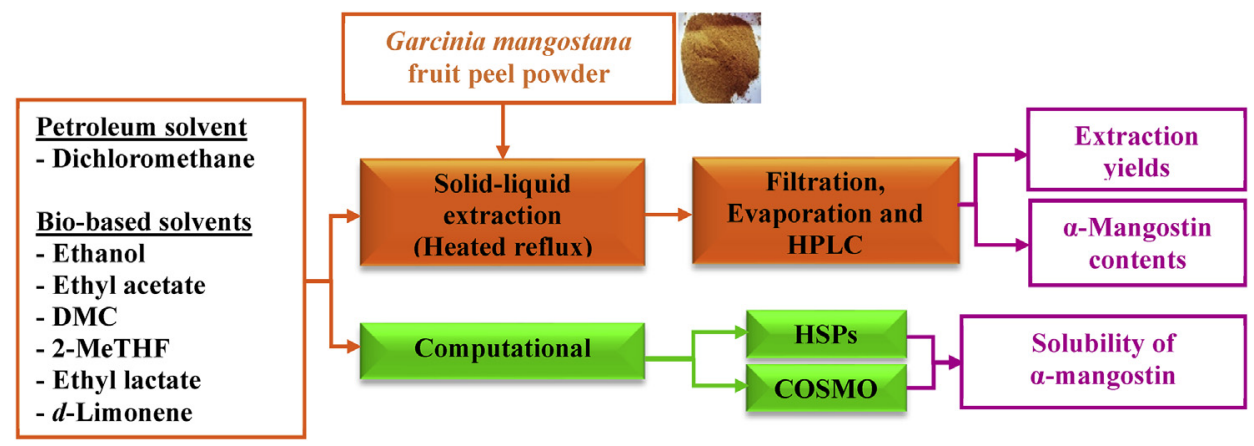

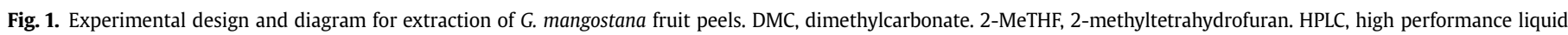
chromatography. HSPs, Hansen solubility parameters. COSMO, conductor like screening model for realistic solvation 
and will dissolve), while medium and poor solvents have RED values of from 1 to 3 and more than 3, respectively. The chemical structures of the solvents and solute (Fig. 2) could be mutually transformed by the JChemPaint version 3.0.1 software, then to their simplified molecular input line entry syntax (SMILES) notations, which were subsequently used for the HSP calculation using the Yamamoto-Molecular Break (Y-MB) method, which can break SMILES into corresponding functional groups and then estimate its HSPs. This method has been embedded in the HSPiP software in order to facilitate the direct HSP calculation for other compounds. These solubility parameters were further modeled to a threedimensional HSP sphere for better visualizing the solute/solvent interactions (HSPiP Version 4.0, Denmark). This method has been embedded in the HSPiP software in order to facilitate the calculation of the HSP of $\alpha$-mangostin and solvents.

\subsection{Computational methods: COSMO-RS}

COSMO-RS uses a statistical thermodynamics approach based on the result of quantum chemical calculations for an understanding of the dissolving mechanism. COSMO-RS combines quantum chemical considerations (COSMO) and statistical thermodynamics (RS) to determine and predict the thermodynamic properties. The COSMO-RS developed by Klamt (2003) is known as a powerful method for molecular description and solvent screening based on a quantum-chemical approach.

COSMO-RS is a two-step procedure including microscopic and macroscopic steps. In the first step, the COSMO model is applied to simulate a virtual conductor environment for the molecule. The molecule is embedded into a virtual conductor (Fig. 3a). In such an environment, the molecule induced a polarization charge density on its surface (Fig. 3b). Therefore, during the quantum calculation self-consistency algorithm the solute molecule is converged to its energetically optimal state in the conductor with respect to its electron density and geometry. The standard quantum chemical methods, triple zeta valence polarized basis set (TZVP) was used in this study. The second step used the statistical thermodynamic calculation. This polarization charge density was used for the quantification of the interaction energy of the pair-wise interacting surface segments with regard to electrostatics and hydrogen bonding. The 3D distribution of the polarization charges on the surface of each molecule was converted into a surface composition function ( $\sigma$-profile) that provided information of the distribution of the molecular polarity (Fig. 3c). The thermodynamics of the

\section{Compound}<smiles>CC(C)=CCc1c(O)cc2oc3cc(C)c(O)c(CC=C(C)C)c3c(=O)c2c1O</smiles>

Solvents

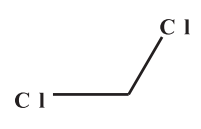

Dichloromethane

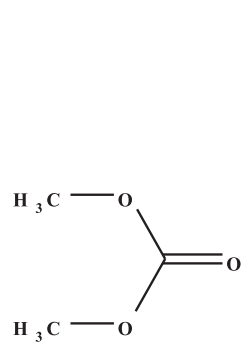

DMC

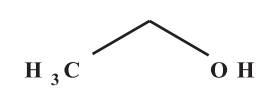

Ethanol<smiles>CC1CCCO1</smiles>

2-MeTHF<smiles>CCOC(=O)C(C)O</smiles>

Ethyl lactate<smiles>CCOC(=O)CC</smiles>

Ethyl acetate<smiles>C=C(C)C1CC=C(C)CC1</smiles>

$d$-Limonene

Fig. 2. Structures of $\alpha$-mangostin and solvents. 
Version définitive du manuscrit publiée dans / Final version of the manuscript published in :

LWT - Food Science and Technology (2016), Vol. 65, p. 297-303, DOI: 10.1016/j.Iwt.2015.08.036

Journal homepage : www.elsevier.com/locate/lwt

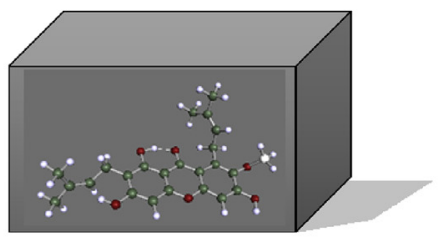

(a) Molecule emerged

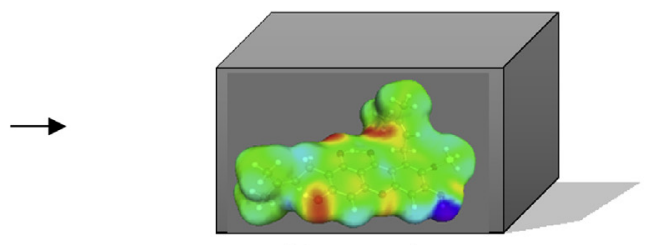

(b) $\sigma$-Surface
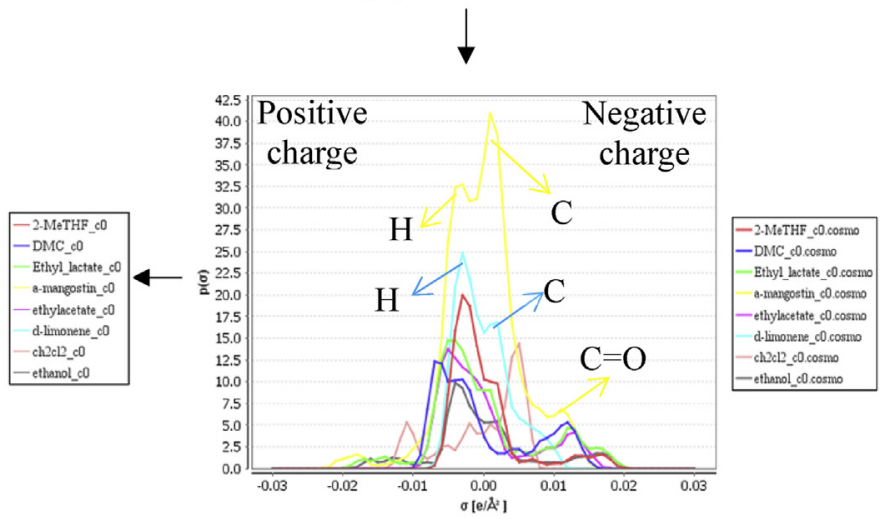

(c) $\sigma$-Profile

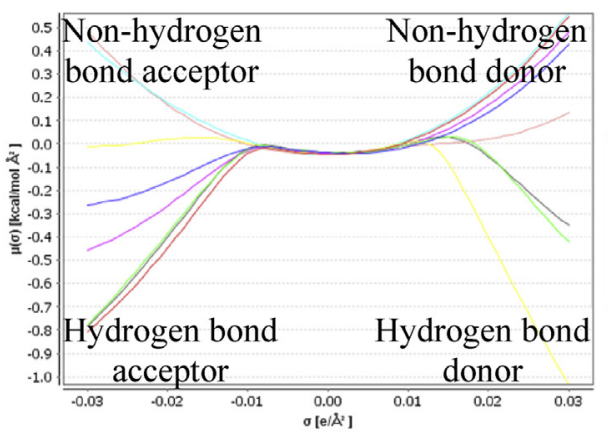

(d) $\sigma$-Potential

$\sigma$-profiles of $\alpha$-mangostin and solvents; (d) $\sigma$ Fig. 3. Step calculation with COSMO-R:
potentials of $\alpha$-mangostin and solvents.

molecular interactions that were based on the obtained $\sigma$-profile was then used to calculate the chemical potential of the surface segment ( $\sigma$-potential) as shown in Fig. 3d using COSMOthermX program (version C30 release 13.01). The $\sigma$-potential described the likeliness of the compound being able to interact with the solvents with polarity and hydrogen bonds (Fig. 3d). The part of the negative charge of the molecule was located on the right side (acceptor hydrogen bonds) with positive $\sigma$ values while the part of the positive charged was located on the left side (donor hydrogen bonds) with negative $\sigma$ values. Generally, the region $\sigma \pm 0.01 \mathrm{e} / \mathrm{A}^{2}$ was considered to be non-polar or weakly polar. The $\sigma$-profile and the $\sigma$-potential were used for interpreting the affinity of the solvent for surface polarity, to understand the interaction between the compound and solvent in mixed state and to estimate the thermodynamic properties of the system.

In addition, the COSMOthermX also calculated the relative solubility between the solid compound and the liquid solvent in terms of the logarithm of the solubility in mole fractions ( $\log 10$ (x_solub)). The logarithm of the best solubility was set to 0 and all other solvents were given relative to the best solvent. The calculation of $\alpha$ mangostin and solvents was performed at the boiling point of each solvent.

\subsection{Solid-liquid extraction}

The dried powders of $G$. mangostana pericarp ( $2 \mathrm{~g}$ ) were sepa- using a rotary vacuum evaporator. The extracts were subjected to HPLC analysis for their $\alpha$-mangostin content. The extraction yields were also recorded. The experiments were performed in triplicate.

\subsection{Quantitative analysis of $\alpha$-mangostin by HPLC}

HPLC analysis of $\alpha$-mangostin was based on the method described by Yodhnu, Sirikatitham, and Wattanapiromsakul (2009) with minor modification. HPLC analysis was carried out using a binary HPLC pump and autosampler (Waters e2695, USA) equipped with a photodiode array detector (Waters 2998, USA). Separation was achieved at $25{ }^{\circ} \mathrm{C}$ on a Lichrocart ${ }^{\circledR}$ column $(5 \mu \mathrm{m}, 250 \mathrm{~mm}$ length $\times 4 \mathrm{~mm}$ internal diameter). The mobile phase consisted of acetonitrile and $0.2 \%$ formic acid in an aqueous solution (70:30; v/ $\mathrm{v})$, and was pumped at a flow rate of $1 \mathrm{~mL} / \mathrm{min}$. The sample injection volume was $20 \mu \mathrm{L}$ and the quantitative detection was by UV at a wavelength of $240 \mathrm{~nm}$. The calibration curve of $\alpha$-mangostin were established using standard $\alpha$-mangostin (concentration range was $12.5,25,50,100$ and $200 \mu \mathrm{g} / \mathrm{mL}$ ). The amount of $\alpha$-mangostin exhibited linearity over the evaluated ranges, with the linear equations of $\mathrm{Y}=78806 \mathrm{X}+78806 \mathrm{X}\left(\mathrm{R}^{2}=1\right)$ respectively. Each calibration point was carried out in triplicate.

The yield of $\alpha$-mangostin was expressed as the weight of $\alpha$ mangostin extracted from the initial G. mangostana powder based on HPLC analysis, as described in Equation (4);

The yield of $\alpha$ - mangostin $=$ weight of $\alpha-$ mangostin/weight of $G$. mangos tan $a$ powder

rately extracted with DMC, 2-MeTHF, ethyl lactate, ethyl acetate, ethanol, $D$-limonene and dichloromethane $(20 \mathrm{~mL})$ under a conventionally heated reflux at the boiling point of each solvent (Table 1 ) for $1 \mathrm{~h}$. The extracts were then filtered and evaporated
The selectivity of each solvent for extraction of $\alpha$-mangostin was expressed from the weight of the $\alpha$-mangostin content in the dried weight of G. mangostana extract, as described in Equation (5): 
Version définitive du manuscrit publiée dans / Final version of the manuscript published in :

LWT - Food Science and Technology (2016), Vol. 65, p. 297-303, DOI: 10.1016/j.Iwt.2015.08.036

Journal homepage : www.elsevier.com/locate/lwt

Selectivity $=$ yield of $\alpha-$ mangostin/weight of dried $G$. mangos $\tan a$ extract

\section{Results and discussion}

\subsection{Solute-solvent solubility by HSPS}

The HSP values of solute and solvents were calculated from their structures (Table 1) and used in plotting a three dimensional (3D) HSP sphere (Fig. 4a) for a theoretical characterization of the solute-solvent interaction. Generally, the optimum HSP for good solvency was characterized by the green dot in the sphere of the 3D graph. However, the result from the HSP showed that all blue dots were outside the sphere to indicate a poor solvency (Fig. 4a). The two dimensional (2D) graph in terms of $\delta_{\mathrm{p}}$ versus $\delta_{\mathrm{h}}$ was plotted for a better understanding of the solubility (Fig. 4b). Moreover, the calculated $R_{a}$ of dichloromethane was less than the other selected solvents (Table 1), showed a center closer to HSP and gave a better solubility for $\alpha$-mangostin than the other solvents. The RED numbers have been used to characterize and quantify the solute-solvent interactions. Table 1 represents the RED modeling optimization of solvents for the extraction of $\alpha$-mangostin. Every solvent showed various theoretical solubility for $\alpha$-mangostin, which was explained by the differences in the solvent polarities.

\subsection{COSMO-RS prediction}

The COSMO-RS data was used to study and understand the potential of alternative solvents for extraction of $\alpha$-mangostin. Analysis of the $\sigma$-profile and the $\sigma$-potential of the components gave some important information about the molecules that could

Table 1

Solvent properties.

\begin{tabular}{|c|c|c|c|c|c|c|c|}
\hline Solvents & Dichloromethane & Ethanol & Ethyl acetate & DMC & 2-MeTHF & Ethyl lactate & D-Limonene \\
\hline \multicolumn{8}{|l|}{ Physicochemical properties } \\
\hline Molecular weight $(\mathrm{g} / \mathrm{mol})$ & 84.93 & 46.07 & 88.11 & 90.07 & 86.13 & 118.14 & 136.23 \\
\hline Density $(\mathrm{g} / \mathrm{mL})$ & 1.25 & 0.78 & 0.90 & 1.00 & 0.86 & 1.04 & 0.83 \\
\hline $\log P$ & 1.19 & -0.31 & 0.79 & 0.15 & 0.82 & -0.04 & 4.45 \\
\hline Flash point $\left({ }^{\circ} \mathrm{C}\right)$ & -14.1 & 8.90 & -3.30 & 18.30 & -11.00 & 48.90 & 48.00 \\
\hline \multicolumn{8}{|l|}{ Toxicities } \\
\hline Resource & Petroleum & Cereal crop & Cereal crop & Chemical synthesis & Cereal crop & Cereal crop & Cereal crop \\
\hline $\mathrm{CMR}^{\mathrm{a}}$ & 2 & No & No & No & No & No & No \\
\hline Toxicity index & 4 & 5 & 5 & 5 & 4 & 5 & 5 \\
\hline \multicolumn{8}{|l|}{ Energy efficiency } \\
\hline Boiling point $\left({ }^{\circ} \mathrm{C}\right)$ & 40 & 73 & 77 & 90 & 80 & 119 & 175 \\
\hline$\delta \mathrm{H}_{\mathrm{vap}}(\mathrm{kJ} / \mathrm{mol})$ & 28.06 & 38.56 & 31.94 & 33.05 & 30.74 & 45.57 & 39.49 \\
\hline $\mathrm{Cp}$, liquid $\left(\mathrm{kJ} / \mathrm{mol}^{*} \mathrm{~K}\right)$ & 0.102 & 0.112 & 0.169 & 0.165 & 0.157 & 0.255 & 0.249 \\
\hline Energy for evaporation of $1 \mathrm{~kg}$ solvent $(\mathrm{kJ})$ & 348.40 & 953.68 & 462.24 & 486.01 & 457.16 & 588.62 & 564.05 \\
\hline \multicolumn{8}{|l|}{ HSPS } \\
\hline$\delta \mathrm{d}\left(\mathrm{MPa}^{1 / 2}\right)$ & 17.4 & 16.2 & 15.7 & 15.9 & 16.4 & 16.6 & 16.7 \\
\hline$\delta \mathrm{p}\left(\mathrm{MPa}^{1 / 2}\right)$ & 8.1 & 8.6 & 5.9 & 8.8 & 4.7 & 7.7 & 1.8 \\
\hline$\delta \mathrm{h}\left(\mathrm{MPa}^{1 / 2}\right)$ & 8.6 & 17.6 & 7.0 & 7.1 & 4.6 & 14.4 & 3.1 \\
\hline$\delta$ Total $\left(\mathrm{MPa}^{1 / 2}\right)$ & 21.1 & 25.4 & 18.2 & 19.5 & 17.6 & 23.2 & 17.1 \\
\hline $\mathrm{Ra}$ & 7.78 & 37.86 & 32.71 & 33.75 & 33.45 & 37.00 & 33.59 \\
\hline RED & 1.94 & 3.10 & 2.31 & 2.63 & 2.19 & 2.40 & 2.22 \\
\hline \multicolumn{8}{|l|}{ COSMO-RS } \\
\hline Log10(x_solub) & 0.16 & 0 & 0 & 0 & 0 & 0 & 0.16 \\
\hline
\end{tabular}

a CMR, carcinogenic, mutagenic and reprotoxic according to European legislation.

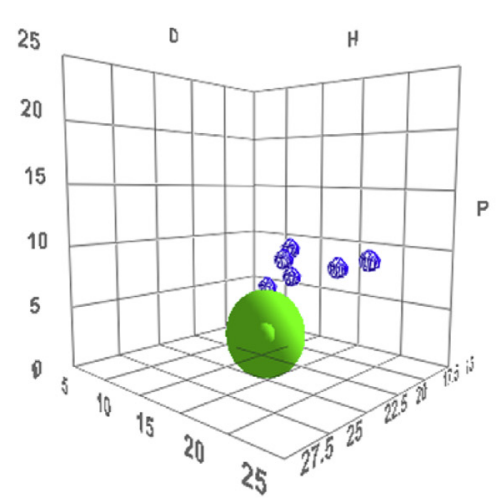

(a)

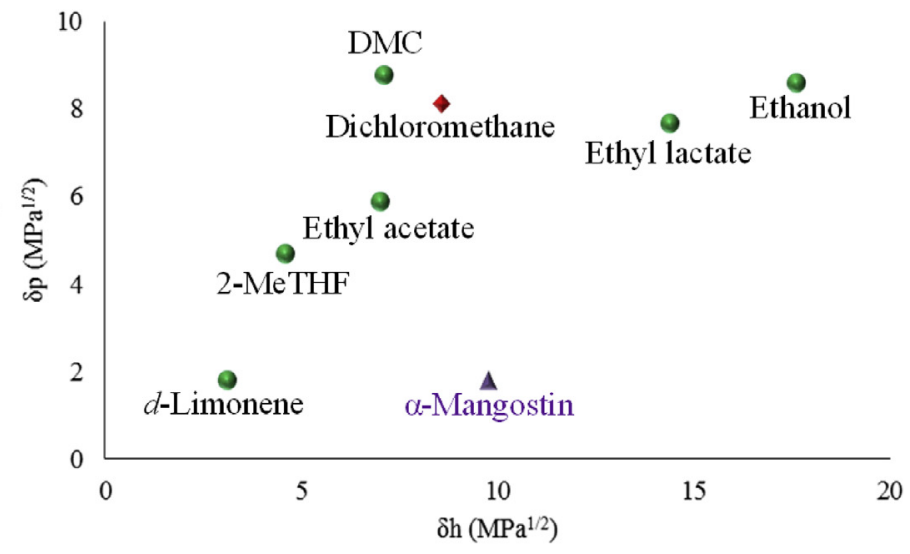

(b)

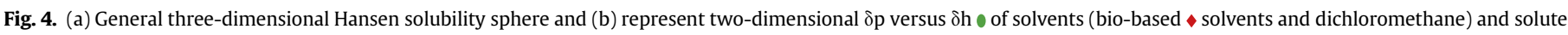
( $\Delta \alpha$-mangostin). 
be used to predict possible interactions in the fluid phase. It was noticed that there was a sign inversion compared to the molecular polarity. For example, the $\sigma$-profile of $\alpha$-mangostin showed three peaks. The first peak in the negatively charged part resulted from the polarized hydrogen atoms, the second peak at a $\sigma$ of $0.005 \mathrm{e} / \mathrm{A}^{2}$ resulted from the carbon atoms and the last peak in the positive charged part resulted from the carbonyl group (Fig. 3c). As a consequence, the $\sigma$-potential was asymmetric due to the electron pairs from the carbonyl group on the negative side (Fig. $3 \mathrm{~d}$ ). The $\sigma-$ profile of $D$-limonene showed two peaks, resulting from the hydrogen atoms on the negative side and from the carbons atoms on the positive side. As a consequence, the $\sigma$-potential of $D$-limonene was similar to the parabola center at $\sigma=0$, a characteristic of a non-polar alkane.

The solubility study between the $\alpha$-mangostin and solvents are written as $\log 10$ (x_solub) (Table 1 ). $\alpha$-Mangostin was more soluble in the solvents that had a $\log 10$ (x_solub) near 0 . The solubility, when compared at the boiling point of each solvent, log10(x_solub) of ethanol, ethyl acetate, ethyl lactate, 2-MeTHF and DMC were close to 0 , unlike dichloromethane and $D$-limonene (Table 1 ). Therefore, $\alpha$-mangostin was more soluble in ethanol, ethyl acetate, ethyl lactate, 2-MeTHF and DMC than in dichloromethane and $D^{-}$ limonene.

\section{3. $\alpha$-Mangostin content and the selectivity of the solvents studied}

The experimental study was performed on G. mangostana pericarp powders using different solvents. The content of $\alpha$-mangostin in each solvent was plotted in Fig. 5. All of the alternative solvents used were capable of extracting $\alpha$-mangostin and gave a higher content of $\alpha$-mangostin than for dichloromethane. The content of $\alpha$-mangostin extracted from the $G$. mangostana powders was in the following order: ethyl lactate $>$ ethanol $>$ DMC $>2$ MeTHF $>$ ethyl acetate $>D$-limonene $>$ dichloromethane. The highest $\alpha$-mangostin content ( $4.19 \mathrm{~g} / 100 \mathrm{~g}$ ) was observed in ethyl lactate. The content was 2 -fold higher than that of the dichloromethane extract $(2.08 \mathrm{~g} / 100 \mathrm{~g})$. The $\alpha$-mangostin contents in the G. mangostana powder were different due to the difference in polarity and solubility between $\alpha$-mangostin and the solvents. The

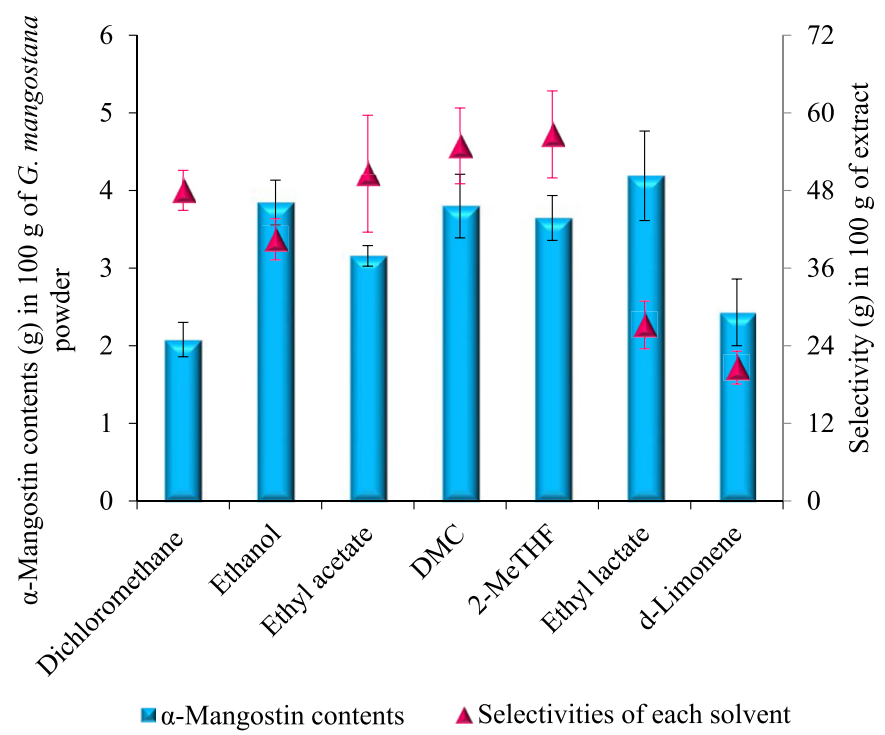

Fig. 5. Quantitative analysis of $\alpha$-mangostin. Data are presented as the mean values of 3 replications \pm SD. results indicated that ethyl lactate was the most suitable solvent for $\alpha$-mangostin extraction.

Furthermore, the $\alpha$-mangostin contents in the dried extracts of each solvent were determined for their selectivity (Fig. 5). The selectivity of each solvent was found in the following order: 2MeTHF $>$ DMC $>$ ethyl acetate $>$ dichloromethane $>$ ethanol $>$ ethyl lactate $>D$-limonene. The highest extractability was obtained with MeTHF (56.68 g/100 g) that was much better than for dichloromethane, ethyl lactate and $D$-limonene. It was different from the previous results that had shown that ethyl lactate gave the highest content of alpha-mangostin extracted from the G. mangostana fruit peel powder.

Therefore, MeTHF, DMC and ethyl acetate had a better selectivity and gave a higher content of $\alpha$-mangostin than dichloromethane. Dichloromethane showed a better selectivity than ethanol, ethyl lactate and $D$-limonene. This was a similar finding to the previous reports on the selectivity of $\alpha$-mangostin extraction using ethanol and dichloromethane (Pothitirat et al., 2010).

However, on further consideration of the energy consumption by these solvents when evaporating $1 \mathrm{~kg}$ of solvent, these alternative solvents consumed more energy than dichloromethane (Table 1). The energy consumption was in the following order: ethanol $>$ ethyl lactate $>D$-limonene $>$ DMC $>$ ethyl acetate $>2$ MeTHF > dichloromethane.

MeTHF, DMC and ethyl acetate were more selective for $\alpha$ mangostin than ethyl lactate and dichloromethane. Dichloromethane showed a higher selectivity than ethanol, ethyl lactate and $D$-limonene. A similar, results had been previously reported using ethanol and dichloromethane (Pothitirat et al., 2010), and this supported the present finding. However, after further consideration of the energy consumption by these solvents when evaporating $1 \mathrm{~kg}$ of solvent, these alternative solvents required more energy than dichloromethane (Table 1 ).

In addition, these solvents were not carcinogenic, mutagenic, and reprotoxic (CMR) compounds. DMC was produced by green organic synthesis (Tundo \& Selva, 2002), 2-MeTHF is a by-product produced from the waste of corn cobs (Antonucci et al., 2011), ethyl acetate is found in rum and grapes damaged by hail or it was produced from ethanol and acetic acid (Filly et al., 2015). Fortunately, all these solvents also showed a higher selectivity for extracting $\alpha$-mangostin than the other solvents. Therefore, DMC, 2MeTHF and ethyl acetate can be promising alternative solvents for the extraction of $\alpha$-mangostin.

\subsection{Comparisons of the experimental, Hansen and COSMO-RS studies}

The experimental data were compared with the results from the predictive modeling: Hansen and COSMO-RS as shown in Table 2.

The Hansen method described dichloromethane as a good solvent for $\alpha$-mangostin extraction, but not according to the experimental data (Table 1). This was mainly because the HSP was reported to have defects in its simulation when studied with nonstandard solvents with large molecules or complex multicomponent systems (Filly et al., 2014).

On other hand, the COSMO-RS method indicated that ethyl lactate, ethanol, DMC, 2-MeTHF and ethyl acetate had a good solubility for $\alpha$-mangostin according to the $\log 10$ (x_solub) values at each boiling point. It was of interest that the results of the COSMORS model correlated well and were consistent with the experimental data. Therefore, the COSMO-RS model was valid for the screening of solvents for $\alpha$-mangostin extraction and allowed for the emerging of bio-based molecules. 
Version définitive du manuscrit publiée dans / Final version of the manuscript published in :

LWT - Food Science and Technology (2016), Vol. 65, p. 297-303, DOI: 10.1016/j.Iwt.2015.08.036

Journal homepage : www.elsevier.com/locate/lwt

Table 2

Comparison of Hansen, COSMO-RS and experimental studies.

\begin{tabular}{|c|c|c|c|}
\hline Solvents & Hansen $\left(\right.$ RED $\left.^{\mathrm{a}}\right)$ & COSMO-RS (log10(x_solub)) & Experiment ( $\alpha$-mangostin content) \\
\hline Dichloromethane & Medium & Low & Low \\
\hline Ethanol & Low & High & High \\
\hline Ethyl acetate & Low & High & High \\
\hline DMC & Low & High & High \\
\hline 2-MeTHF & Low & High & High \\
\hline Ethyl lactate & Low & High & High \\
\hline$D$-Limonene & Low & Low & Low \\
\hline
\end{tabular}

${ }^{a}$ RED, relative energy difference.

\section{Conclusion}

Application of the predictive COSMO-RS model was better suited than the Hansen model for selecting alternative solvents for extracting $\alpha$-mangostin. The experimental study confirmed that the COSMO-RS simulation was more suitable for screening the solvent for complex molecules. From this study it was concluded that DMC, 2-MeTHF and ethyl acetate were promising green solvents for the extraction of bioactive compounds involved with the pharmaceutical industry, with high extraction efficacy, high selectivity, the saving of time and energy as well as being an economically viable and environmentally friendly approach.

\section{Acknowledgments}

The authors wish to thank Prince of Songkla University and Royal Golden Jubilee scholarship (Grant No. PHD/0115/2554) for financial support of this research. Also thanks to Dr. Brian Hodgson for assistance with the English.

\section{References}

Antonucci, V., Coleman, J., Ferry, J. B., Johnson, N., Mathe, M., Scott, J. P., et al. (2011). Organic Process Research \& Development, 15, 939-941.

CalEPA (California environmental protection agency). (2000). Public health goals for chemicals in drinking water: Dichloromethane (methylene chloride, DCM). Available http://oehha.ca.gov/water/phg/pdf/dcm.pdf (accessed 09.04.15).

Cui, J., Hu, W., Cai, Z., Liu, Y., Li, S., Tao, W., et al. (2010). New medicinal properties of mangostins: analgesic activity and pharmacological characterization of active ingredients from the fruit hull of Garcinia mangostana L. Pharmacology Biochemistry and Behavior, 95, 166-172.

Filly, A., Fabiano-Tixier, A. S., Fernandez, X., \& Chemat, F. (2015). Alternative solvents for extraction of food aromas. Experimental and COSMO-RS study. LWT - Food Science and Technology, 61, 33-40 (The authors performs comparative study using COSMO-RS and experimental for selection of alternative solvents to replace hexane).

Filly, A., Fabiano-Tixier, A. S., Lemasson, Y., Roy, C., Fernandez, X., \& Chemat, F. (2014). Extraction of aroma compounds in blackcurrant buds by alternative solvents: theoretical and experimental solubility study. Comptes Rendus Chimie, 17, 1268-1275 (Demonstration of methodology for Hansen solubility parameter model).

Hansen, C. M. (1969). The universality of the solubility parameter. Industrial and
Engineering Chemistry Product Research and Development, 8, 2.

Klamt, A. (2003). Prediction of the mutual solubilities of hydrocarbons and water with COSMO-RS. Fluid Phase Equilibria, 206, 223-235.

Li, Y., Fabiano-Tixier, A. S., Ginies, C., \& Chemat, F. (2014). Direct green extraction of volatile aroma compounds using vegetable oils as solvents: theoretical and experimental solubility study. LWT - Food Science and Technology, 59, 724-731 (Demonstration of methodology for Hansen solubility parameter model.).

Moongkarndi, P., Kosem, N., Kaslungka, S., Luanratana, O., Pongpan, N., \& Neungton, N. (2004). Antiproliferation, antioxidation and induction of apoptosis by Garcinia mangostana (mangosteen) on SKBR3 human breast cancer cell line. Journal of Ethnopharmacology, 90, 161-166.

Nganlasom, J., Suttitum, T., Jirakulsomchok, D., \& Puapairoj, A. (2008). Effects of Centella asiatica Linn. leaves and Garcinia Mangostana Linn. hull on the healing of dermal wounds in diabetic rats. Srinagarind Medical Journal, 23, 402-407.

Nualkaew, N., Morita, H., Shimokawa, Y., Kinjo, K., Kushiro, T., De-Eknamkul, W. et al. (2012). Benzophenone synthase from Garcinia mangostana L. pericarps. Phytochemistry, 77, 60-69.

Pothitirat, W., Chomnawang, M. T., \& Gritsanapan, W. (2009). Anti-acne inducing bacteria activity and $\alpha$-mangostin content of Garcinia mangostana fruit rind extracts from different provenience. Songklanakarin Journal of Science and Technology, 31, 41-47.

Pothitirat, W., Chomnawang, M. T., \& Gritsanapan, W. (2010). Anti-acne-inducing bacterial activity of mangosteen fruit rind extracts. Medical Principles and Practice, 19, 281-286. Optimization of suitable solvents for extraction of $\alpha$ mangostin.

Rombaut, N., Fabiano-Tixier, A. S., Bily, A., \& Chemat, F. (2014). Green extraction processes of natural products as tools for biorefinery. Biofuels, Bioproducts and Biorefining, 8, 530-544.

Sakagami, Y., Iinuma, M., Piyasena, K., \& Dharmaratne, H. (2005). Antibacterial activity of $\alpha$-mangostin against vancomycin resistant enterococci (VRE) and synergism with antibiotics. Phytomedicine, 12, 203-208.

Sicaire, A. G., Vian, M., Fine, F., Joffre, F., Carre, P., Tostain, S., et al. (2015). Alternative bio-based solvents for extraction of fat and oils: solubility prediction, global yield, extraction kinetics, chemical composition and cost of manufacturing. International Journal of Molecular Sciences, 16, 8430-8453.

Tanzi, C. D., Abert-Vian, M., Ginies, C., Elmaataoui, M., \& Chemat, F. (2012). Terpenes as green solvents for extraction of oil from microalgae. Molecules, 17, 8196-8205.

Tundo, P., \& Selva, M. (2002). The chemistry of dimethylcarbonate. Accounts of Chemical Research, 35, 706-716.

Yodhnu, S., Sirikatitham, A., \& Wattanapiromsakul, C. (2009). Validation of LC for the determination of $\alpha$-mangostin in mangosteen pericarp extract: a tool for quality assessment of Garcinia mangostana L. Journal of Chromatographic Science, 47, 185-189 (Demonstration of HPLC quantitative analysis for $\alpha$-mangostin).

Zarena, A. S., \& Sankar, K. U. (2011). Xanthones enriched extracts from mangosteen pericarp obtained by supercritical carbon dioxide process. Separation and $\mathrm{Pu}$ rification Technology, 80, 172-178. 